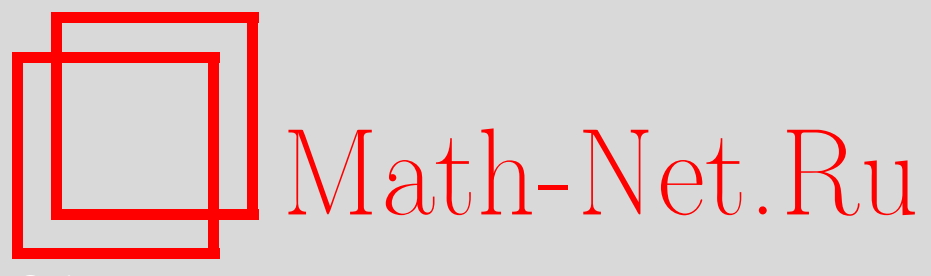

С. А. Имомкулов, З. Ш. Ибрагимов, Плюриполярность графиков квазианалитических функций в смысле Гончара, Матем. заметки, 2011, том 89, выпуск 4, 637-640

DOI: https://doi.org/10.4213/mzm8643

Использование Общероссийского математического портала Math-Net.Ru подразумевает, что вы прочитали и согласны с пользовательским соглашением http://www.mathnet.ru/rus/agreement

Параметры загрузки:

IP : 18.209 .158 .208

26 апреля 2023 г., 04:57:19

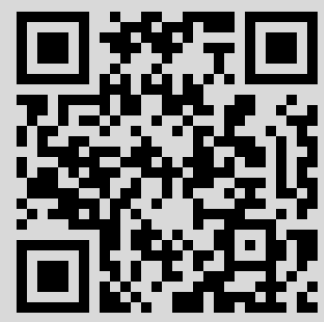




\section{Плюриполярность графиков квазианалитических функций в смысле Гончара}

\section{С. А. Имомкулов, З. Ш. Ибрагимов}

Пусть $f$ - функция, определенная и непрерывная на отрезке $\Delta=[a, b]$ действительной прямой $\mathbb{R}, \rho_{n}(f)$ - наименьшее отклонение функции $f$ на отрезке $\Delta$ от рациональных функций степени не выше $n$ :

$$
\rho_{n}(f)=\inf _{\left\{r_{n}\right\}}\left\|f-r_{n}\right\|_{\Delta},
$$

где $\|\cdot\|_{\Delta}-$ равномерная норма и нижняя грань берется в классе всех рациональных функций вида

$$
r_{n}(x)=\frac{a_{0} x^{n}+a_{1} x^{n-1}+\cdots+a_{n}}{b_{0} x^{n}+b_{1} x^{n-1}+\cdots+b_{n}} .
$$

Как обычно, через $e_{n}(f)$ обозначим наименьшее отклонение функции $f$ на $\Delta$ от полиномов степени $\leqslant n$. Очевидно, $\rho_{n}(f) \leqslant e_{n}(f)$ при любом $n=0,1,2, \ldots$.

В работах Гончара [1], [2] доказано, что класс функций

$$
R(\Delta)=\left\{f \in C(\Delta): \varliminf_{n \rightarrow \infty} \sqrt[n]{\rho_{n}(f)}<1\right\}
$$

обладает одним из важнейших свойств класса аналитических функций: если

$$
\varliminf_{n \rightarrow \infty} \sqrt[n]{\rho_{n}(f)}<1
$$

и $f(x)=0$ на множестве $E \subset \Delta$ положительной логарифмической емкости, то $f(x) \equiv 0$, $x \in \Delta$.

По аналогии с классом

$$
B(\Delta)=\left\{f \in C(\Delta): \varliminf_{n \rightarrow \infty} \sqrt[n]{e_{n}(f)}<1\right\}
$$

который называется классом квазианалитических функиий Бернштейна (см. [3]), класс $R(\Delta)$ мы назовем классом квазианалитических функиий Гончара.

Хорошо известно, что функции, аналитические на отрезке $\Delta$, характеризуются условиями

$$
\varlimsup_{n \rightarrow \infty} \sqrt[n]{e_{n}(f)}<1
$$

(теорема Бернштейна). Отсюда следует, что класс аналитических на отрезке $\Delta$ функций $A(\Delta)$ является подклассом классов $B(\Delta) \subset R(\Delta)$, т.е. $A(\Delta) \subset B(\Delta) \subset R(\Delta)$. Ясно, что $A(\Delta) \neq B(\Delta)$. В работе Гончара [1] показано, что существуют функции, для которых отклонения при приближении полиномами $e_{n}(f)$ стремятся к нулю сколь угодно медленно, в то время как $\rho_{n}(f)$ стремятся к нулю сколь угодно быстро. Отсюда, в частности вытекает, что

$$
B(\Delta) \neq R(\Delta) .
$$

Нетрудно убедится в том, что если $f \in A(\Delta)$, то ее график

$$
\Gamma_{f}=\left\{(x, f(x)) \in \mathbb{C}^{2}: x \in \Delta\right\}
$$

является плюриполярным множеством в $\mathbb{C}^{2}$. В работе [4] Дидрих и Форнесс построили пример бесконечно дифференцируемой функции, график которой не является плюриполярным в $\mathbb{C}^{2}$. В недавней работе Комана, Левенберга и Полецкого [5] доказано, что если $f \in B(\Delta)$, то ее график $\Gamma_{f}$ является плюриполярным в $\mathbb{C}^{2}$. 
Мы докажем следующую более общую теорему.

Tеорема. Если $f \in R(\Delta)$, то ее график $\Gamma_{f}$ плюриполярен в $\mathbb{C}^{2}$.

ДокАзАтЕЛЬСтво. Согласно условию теоремы существует последовательность натуральных чисел $n_{k}$ и соответствующая последовательность рациональных функций

$$
r_{n_{k}}=\frac{p_{n_{k}}}{q_{n_{k}}}
$$

такие, что

$$
\rho_{n_{k}}(f)=\left\|f-r_{n_{k}}\right\|_{\Delta} \leqslant \alpha^{n_{k}},
$$

где $\alpha$ - некоторое фиксированное число, $0<\alpha<1$. Не нарушая общности, мы можем предполагать, что

$$
\|f\|_{\Delta} \leqslant \frac{1}{2}, \quad\left\|p_{n_{k}}\right\|_{\Delta} \leqslant 1, \quad\left\|q_{n_{k}}\right\|_{\Delta}=1 .
$$

Согласно неравенству Бернштейна- Уолша (см. [6])

$$
\left|p_{n_{k}}(z)\right| \leqslant e^{n_{k} V(z, \Delta)}, \quad\left|q_{n_{k}}(z)\right| \leqslant e^{n_{k} V(z, \Delta)}
$$

для любых $z \in \mathbb{C}$ и $k \in \mathbb{N}$. Здесь $V(z, \Delta)$ - функция Грина дополнения к отрезку $\Delta$ с полюсами в $\infty$ (она равна $\ln \left|z+\sqrt{z^{2}-1}\right|$, если $\left.[a, b]=[-1,1]\right)$.

Введем следующую вспомогательную последовательность плюрисубгармонических функций:

$$
u_{k}(z, w)=\frac{1}{n_{k}} \ln \left|q_{n_{k}}(z) \cdot w-p_{n_{k}}(z)\right|, \quad(z, w) \in \mathbb{C}^{2} .
$$

Имеем

$$
\begin{aligned}
& \frac{1}{n_{k}} \ln \left|q_{n_{k}}(z) \cdot w-p_{k}(z)\right| \leqslant \frac{1}{n_{k}} \ln \left(\left|q_{n_{k}}(z) \cdot w\right|+\left|p_{n_{k}}(z)\right|\right) \\
& \quad \leqslant \max \left\{\frac{1}{n_{k}} \ln 2\left|p_{k}(z)\right|, \frac{1}{n_{k}} \ln 2\left|q_{n_{k}}(z) \cdot w\right|\right\} \\
& \quad=\max \left\{\frac{1}{n_{k}} \ln \left|p_{k}(z)\right|, \frac{1}{n_{k}} \ln \left|q_{n}(z)\right|+\frac{1}{n_{k}} \ln |w|\right\}+\frac{\ln 2}{n_{k}}, \quad(z, w) \in \mathbb{C}^{2} .
\end{aligned}
$$

Отсюда мы получим оценку

$$
u_{k}(z, w) \leqslant \max \left\{V(z, \Delta), V(z, \Delta)+\frac{1}{n_{k}} \ln |w|\right\}+\frac{\ln 2}{n_{k}} .
$$

Следовательно, последовательность плюрисубгармонических функций $u_{k}(z, w)$ локально равномерно ограничена сверху. Пусть

$$
u(z, w)=\varlimsup_{k \rightarrow \infty} u_{k}(z, w) .
$$

Функция $u(z, w)$ также локально ограничена сверху,

$$
u(z, w) \leqslant V(z, \Delta) .
$$

Обозначим через

$$
u^{*}(z, w)=\varlimsup_{\left(z^{\prime}, w^{\prime}\right) \rightarrow(z, w)} u\left(z^{\prime}, w^{\prime}\right)
$$

регуляризацию функции $u(z, w)$. Множество

$$
E=\left\{(z, w) \in \mathbb{C}^{2}: u(z, w)<u^{*}(z, w)\right\}
$$

является плюриполярным в $\mathbb{C}^{2}$ (см. [6], [7]). 
Пусть теперь $(z, w) \in \Gamma_{f}$ - фиксированная точка. Тогда (отметим, что $q_{n}(z) \neq 0$ при $z \in \Delta)$

$$
\begin{aligned}
u(z, w) & =\varlimsup_{k \rightarrow \infty} \ln \left|q_{n_{k}}(z)\right|^{1 / n_{k}}\left|w-\frac{p_{n_{k}}(z)}{q_{n_{k}}(z)}\right|^{1 / n_{k}} \leqslant \varlimsup_{k \rightarrow \infty} \ln \alpha\left|q_{n_{k}}(z)\right|^{1 / n_{k}} \\
& =\ln \alpha+\varlimsup_{k \rightarrow \infty} \ln \left|q_{n_{k}}(z)\right|^{1 / n_{k}} .
\end{aligned}
$$

Если же $(z, w) \in(\Delta \times \mathbb{C}) \backslash \Gamma_{f}$, то

$$
\begin{aligned}
u(z, w) & =\varlimsup_{k \rightarrow \infty} \frac{1}{n_{k}} \ln \left|q_{n_{k}}(z) w-p_{n_{k}}(z)\right|=\varlimsup_{k \rightarrow \infty} \ln \left|q_{n_{k}}(z)\right|^{1 / n_{k}}\left|w-\frac{p_{n_{k}}(z)}{q_{n_{k}}(z)}\right|^{1 / n_{k}} \\
& =\varlimsup_{k \rightarrow \infty} \ln \left|q_{n_{k}}(z)\right|^{1 / n_{k}} .
\end{aligned}
$$

Отсюда вытекает, что если в точке $z \in \Delta$

$$
\varlimsup_{k \rightarrow \infty}\left|q_{n_{k}}(z)\right|^{1 / n_{k}} \neq 0,
$$

то $(z, f(z))$ принадлежит плюриполярному множеству $E$. Поэтому для завершения доказательства теоремы достаточно установить, что множество

$$
A=\left\{z \in \Delta: \varlimsup_{k \rightarrow \infty}\left|q_{n_{k}}(z)\right|^{1 / n_{k}}=0\right\}=\left\{z \in \Delta: \lim _{k \rightarrow \infty}\left|q_{n_{k}}(z)\right|^{1 / n_{k}}=0\right\}
$$

полярно.

Положим

$$
\vartheta_{k}(z)=\sup _{m \geqslant k} \ln \left|q_{n_{m}}(z)\right|^{1 / n_{m}}, \quad \vartheta_{k}^{*}(z)=\varlimsup_{z^{\prime} \rightarrow z} \vartheta_{k}\left(z^{\prime}\right), \quad z \in \mathbb{C} .
$$

Тогда $F_{k}=\left\{\vartheta_{k}(z)<\vartheta_{k}^{*}(z)\right\}, k \in \mathbb{N},-$ полярные множества. Субгармонические функции $\vartheta_{k}^{*}(z)$ монотонно убывают и сходятся к $-\infty$ на $A \backslash \bigcup_{k} F_{k}$.

Так как $\left\|q_{n_{k}}\right\|_{\Delta}=1$, то отсюда легко вытекает, что функция $\lim _{k \rightarrow \infty} \vartheta_{k}^{*}(z)$ не равна тождественно $-\infty$. Таким образом, множество $A \backslash \bigcup_{k} F_{k}$ полярное и, следовательно, $A$ является полярным множеством. Теорема доказана.

Добавление А. Садуллаева. В работе, на самом деле, доказано больше: пусть $K$ произвольный компакт в пространстве $\mathbb{C}^{n}$ и $f(z) \in C(K)$. Мы будем говорить, что $f(z)$ принадлежит классу Гончара $R(K)$, если

$$
\varliminf_{k \rightarrow \infty} \rho_{k}^{1 / k}(f, K)<1, \quad \rho_{k}(f, K)=\inf _{\left\{r_{k}\right\}}\left\|f-r_{k}\right\|_{K},
$$

где inf берется по всем рациональным функциям $r_{k}(z)$ степени $\leqslant k$. Из проведенных выше рассуждений вытекает следующая более общая теорема: если функция $f(z)$ принадлежит классу Гончара $R(K)$, то ее график $\Gamma_{f}=\left\{(z, f(z)) \in \mathbb{C}^{n+1}: z \in K\right\}$ плюриполярен в $\mathbb{C}^{n+1}$.

Отметим, что плюриполярность графика $\Gamma_{f}$ тесно связана с аналитичностью или с близкими к аналитичности свойствами функции $f(z)$. В работе Щербины [8] доказана аналитичность непрерывной в области $D \subset \mathbb{C}^{n}$ функции $f(z)$, график которой плюриполярен в $\mathbb{C}^{n+1}$. В работе [9] построена голоморфная в единичном круге $U \subset \mathbb{C}$ и непрерывная вплоть до границы функция $f(z)$, график

$$
\Gamma_{f}=\left\{(z, f(z)) \in \mathbb{C}^{2}: z \in \bar{U}\right\},
$$

которой не плюриполярен. Все это наводит на мысль, что для плюриполярности графиков главную роль играет свойство аналитичности или квазианалитичности функции. Тем более, в работах [5], [4] доказано, что квазианалитичность в смысле Донжуа также влечет за собой плюриполярность графика. 
В работе [10] приведен пример лакунарного ряда

$$
f(z)=\sum_{k=1}^{\infty} a_{n_{k}} z^{n_{k}}
$$

$\left(\varlimsup_{i m} \sqrt{n_{k}}{ }_{a_{n_{k}}}=1, n_{k} / n_{k+1} \rightarrow 0, k \rightarrow \infty\right)$ такого, что график $\Gamma_{f}$ функции $f$ плюриразрежен в каждой граничной точке. Интересно было бы установить, является ли замыкание графиков бесконечно гладкой вплоть до границы функции

$$
f(z)=\sum_{k=1}^{\infty} \frac{1}{(k !)^{k}} z^{k !}
$$

плюриполярной в $\mathbb{C}^{2} ?$ Если рассмотреть ряд

$$
f(z)=\sum_{k=1}^{\infty} \frac{1}{2^{(k-1) !}} z^{k !},
$$

то он будет плюриполярным, ибо $f(z)$ в замкнутом единичном круге квазианалитична в смысле Гончара.

\section{СПИСОК ЦИТИРОВАННОЙ ЛИТЕРАТУРЫ}

[1] А. А. Гончар, Докл. АН СССР, 100:2 (1955), 205-208. [2] А. А. Гончар, Изв. АН АрмССР. Матем., 6:2-3 (1971), 148-159. [3] A.F. Timan, Theory of Approximation of Functions of a Real Variable, Internat. Ser. Monogr. Pure Appl. Math., 34, Pergamon Press, New York, 1963. [4] K. Diederich, J. E. Fornass, Duke Math. J., 49:4 (1982), 931-936. [5] D. Coman, N. Levenberg, E. A. Poletsky, J. Amer. Math. Soc., 18:2 (2005), 239-252. [6] А. Садуллаев, Комплексный анализ - многие переменные - 2, Итоги науки и техн. Сер. Соврем. пробл. мат. Фундам. направления, 8, ВИНИТИ, М., 1985, 65-113. [7] А. С. Садуллаев, Матем. сб., 119:1 (1982), 96-118. [8] N. Shcherbina, Acta Math., 194:2 (2005), 203-216. [9] N. Levenberg, G. Martin, E. A. Poletskiy, Indiana Univ. Math. J., 41:2 (1992), 515-532. [10] А. С. Садуллаев, УMH, 36:4 (1981), 53-105.

\section{С. А. Имомкулов}

Навоийский государственный педагогический институт, Узбекистан

E-mail: sevdi@rambler.ru

\section{З. Ш. Ибрагимов}

Ургенчский государственный университет им. Аль-Хорезми, Узбекистан

E-mail: z.ibragim@gmail.com
Поступило

10.11.2009

Исправленный вариант

11.06.2010 